\title{
Prosthetic Rehabilitation of Amputated Fingers Using Thimble Prosthesis: A Novel Methodology
}

\author{
Sumedha Dewan ${ }^{1}$ Tarun Kalra ${ }^{1}$ Manjit Kumar ${ }^{1} \quad$ Ajay Bansal ${ }^{1}$ \\ 1Department of Prosthodontics, Bhojia Dental College and Hospital, \\ Himachal Pradesh, India

\begin{abstract}
Address for correspondence Sumedha Dewan, Department of Prosthodontics, Bhojia Dental College and Hospital, Village Budh, Tehsil Baddi, Solan 173205, Himachal Pradesh, India (e-mail: sumedha.dewan09@gmail.com).
\end{abstract}

Dent J Adv Stud 2019;7:131-134

\begin{abstract}
Keywords

- silicone elastomers

- thimble prosthesis

- traumatic injury

- amputation

Any kind of deformity or injury to the finger or hand is one of the most encountered type of injuries, which may cause psychological and emotional disturbance to the patient. The prosthetic rehabilitation of the amputed part of the finger supports the patient emotionally as well as physically and also has a role in making the patient socially more acceptable. This case report highlights on a simple technique for fabricating a thimble prosthesis using silicone elastomers.
\end{abstract}

\section{Introduction}

The most common situations which demand for the fabrication of finger prosthesis are amputations due to traumatic injuries, congenitally missing situations, or malformations. Loss of fingers can result in compromised grasping capacity, emotional and psychological trauma to the patient, and requires serious attention. ${ }^{1} \mathrm{~A}$ thimble type prosthesis offers the advantage over full length finger prosthesis that (1) it allows unrestricted movement of proximal inter-phalangeal joint and (2) minimizes coverage and allows maximum sensation of the intact skin. ${ }^{2}$

There are various types of amputations like self-amputation which results when the trapped person tries to free himself/herself by removing a part of his body and traumatic amputation which can be a result of any accident in factory, farm, with powered tools or motor vehicle accidents. ${ }^{3}$

Ideal finger prosthesis should have a natural looking appearance with surface details, maximum skin shade matching, thin margins which should merge with the skin, incorporating characterization features like fingernails, and reproducing wrinkles and pigmentations. The anatomy of the residual stump is of utmost importance and will perceive the mode of retention to be used, and the level of esthetics one can expect. An ideal residual stump should have adequate length, compressibility, and contours that will provide excellent esthetics and good retention. However, this may not be encountered in all cases that we come across. This case report describes a cost effective and novel technique of

published online January 20, 2020
DOI https://doi.org/

$10.1055 / \mathrm{s}-0039-3402303$

ISSN 2321-1482. rehabilitation of partially amputated fingers with bulbous distal anatomy by fabricating a thimble prosthesis using silicone for a patient who lost his finger using a powered tool.

\section{Case Report}

A 56-year-old male patient, carpenter by profession reported to the Department of Prosthodontics and crown and bridge for rehabilitation of partially amputated middle and ring finger of left hand. History revealed that the amputation was done following an accident with powered tool at his work shop. The stumps showed a thickened end with no signs of infection ( - Fig. 1). After clinical evaluation of the defect it was concluded that the amputated stumps were acceptable for prosthetic rehabilitation. The patient was explained about the technique and convinced for the rehabilitation of missing fingers with a thimble type of prosthesis.

\section{Making the Impression}

Impression of the traumatized hand was made with irreversible hydrocolloid (Zelgan 2002, Dentsply, Gurgaon, Haryana). A thin layer of petroleum jelly was applied to the area to avoid adherence of impression material to hair and skin. A suitable plastic container having adequate clearance of at least $5 \mathrm{~mm}$ all around and sufficient length to cover the hand was selected for the impression material. Cold water was used to manipulate alginate to increase the working time and poured into the plastic container. The
License terms

Hospital af

Pradesh University
() (1) $\ominus \circledast$ 
patient's hand was dipped vertically into the container with fingers apart taking care not to touch the walls of the container. After the material was set the patient was asked to carefully remove his hand with a jerk (-Fig. 2). The impression was poured using dental stone (Kalabhai Stone Plaster Kalstone, Maharashtra). ${ }^{4}$ Then an impression of the patient's right hands middle and ring finger was made in a similar way to use it as a guide to carve and make wax pattern of the missing fingers using modelling wax (Rolex, Modelling Wax No.2, Ashoo Sons, New Delhi; -Fig. 3).

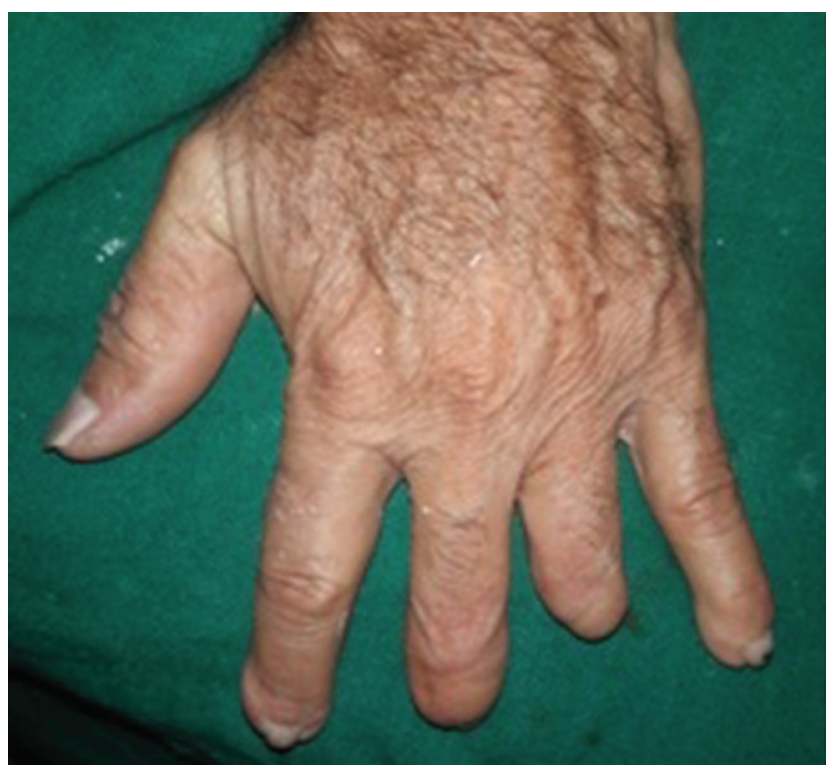

Fig. 1 Amputee fingers.

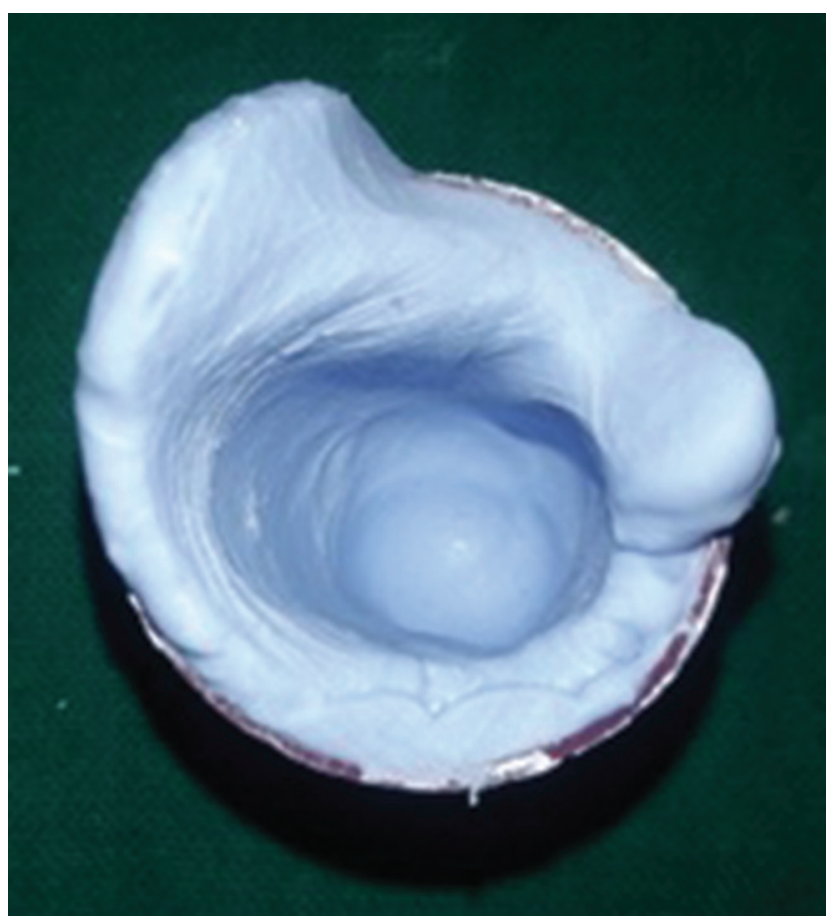

Fig. 2 Alginate impression of the amputated finger made.

\section{Preparing the Stump}

The stone model was trimmed $0.5 \mathrm{~mm}$ circumferentially smaller than the segment of the stump over which prosthesis will "cup" to achieve a good suction fit. The problem is worsened by the fact that the suction fitted prosthesis acts very much like a pressure garment and when worn for a prolonged period can shrink the residuum by soft tissue compression. $^{2}$

The percentage of circumferential reduction of the positive models of the residuum is calculated by the equation ${ }^{2}$ :

Percentage of circumferential $=$ residuum

model (circumference) - finger model(circumference) $\times 100 \%$ Residuum model(circumference)

\section{Reduction of Finger Model}

A 5 to $7 \%$ circumferential reduction in the finger model proves to be a good fit for thimble type prosthesis for distal finger amputations. $^{2}$

\section{Try in of Wax Pattern}

The wax pattern of the finger of other hand was hollowed by carving out the wax and placed and adapted on the circumferentially reduced amputated finger model. During this stage the length, shape, and fit of the wax pattern was verified and checked for harmony with adjacent fingers (-Figs. 4 and 5). Necessary modifications were made at this stage to improve the adaptation and the anatomy was also checked and refined to improve esthetics. ${ }^{4}$

An artificial nail with cold cure acrylic resin was fabricated $^{5}$ and finished and polished (-Fig. 6).

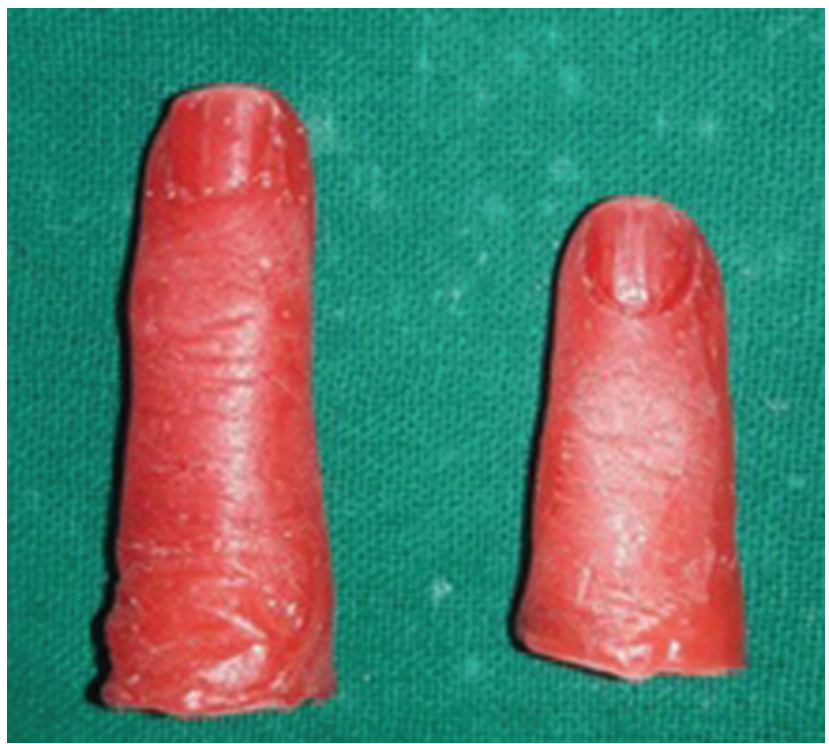

Fig. 3 Wax models obtained from alginate impression of other hand. 
The pattern and the cast were invested in the flask such that the dorsal half of the pattern was invested separately. Tin foil substitute was applied and then ventral half was poured. Wax was eliminated in a conventional way.

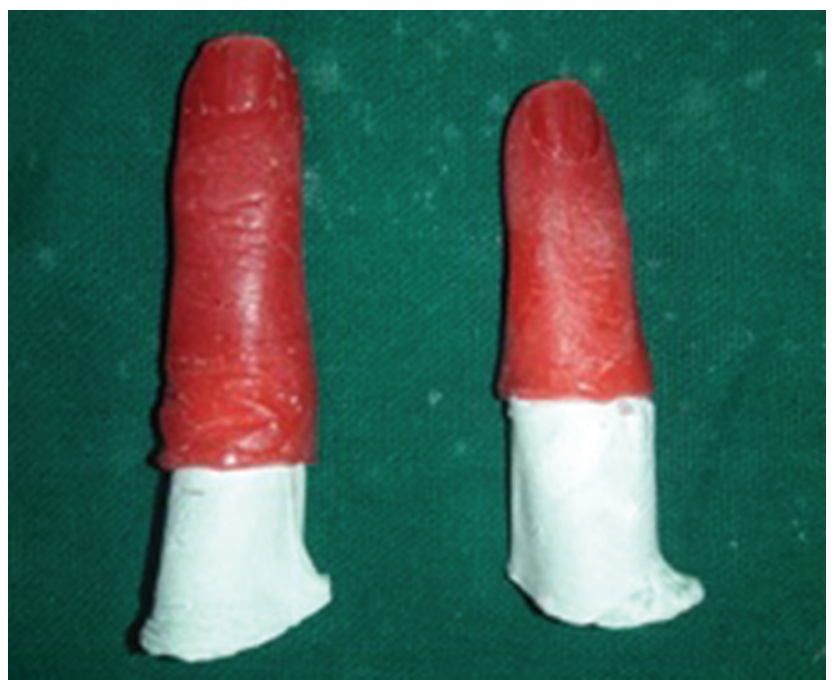

Fig. 4 Wax patterns fitted on the prepared stumps.

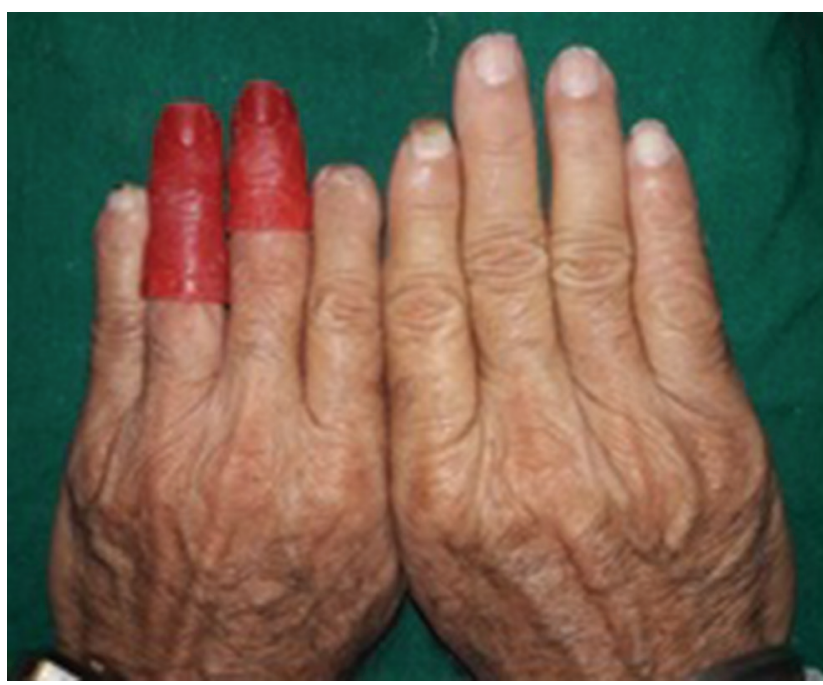

Fig. 5 Fitting of wax pattern checked on the patient.

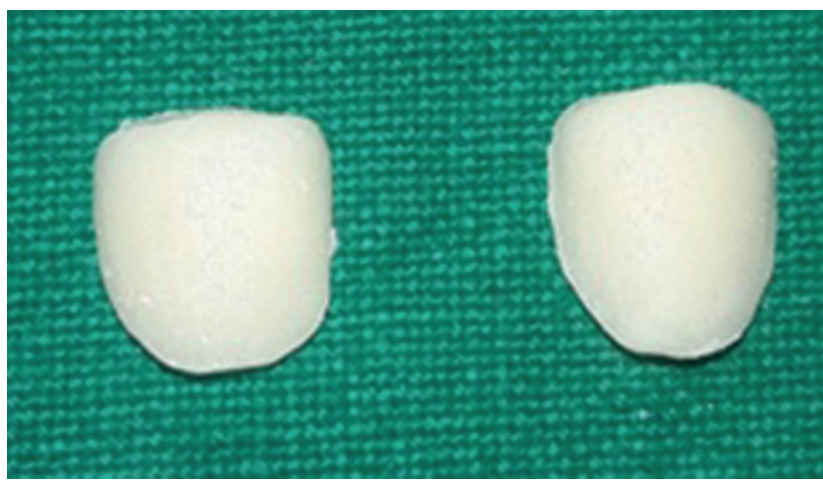

Fig. 6 Artificial nails fabricated from clear acrylic.

\section{Color Matching and Incorporation of Nail}

Room temperature vulcanizing silicone (Dragon Skin, Smooth on Inc., PA) was intrinsically pigmented according to the skin tone of the patient ( - Fig. 7 ). The base color of the skin was observed and maximum efforts were made to achieve the desired shade. Shade matching for the dorsal and ventral surface was done separately under natural light. ${ }^{1}$

The nail, which was fabricated using acrylic resembling the size and shape of the nails of the other hand to allow a natural appearance, was then incorporated into the space of the nail bed in the mold obtained after dewaxing. The mold was then packed with silicone material both in the dorsal and ventral half and allowed to bench cure overnight ( $\sim$ Fig. 8). It is the property of the material that it gets dense when $1 \mathrm{~A}: 1 \mathrm{~B}$ are mixed and they blend only at a thin stream line which is produced at the junction of the dorsal and ventral half when the die is packed with the silicone material.

After retrieval of the prosthesis the excess was trimmed with a scalpel or scissors and finished and polished. The size and position of the acrylic nail (DPI Cold cure, The Bombay Burmah Trading Corporation Ltd., Mumbai) was then secured using cyanoacrylate adhesive under its surface for bonding with silicone. The fit and the appearance of the prosthesis was checked and the patient was instructed about the maintenance of the prosthesis. ${ }^{3}$ Homecare instructions involving the use of a soft brush, soap, and warm water irrigation were given, and the patient was instructed to come back after 2 months for a recall

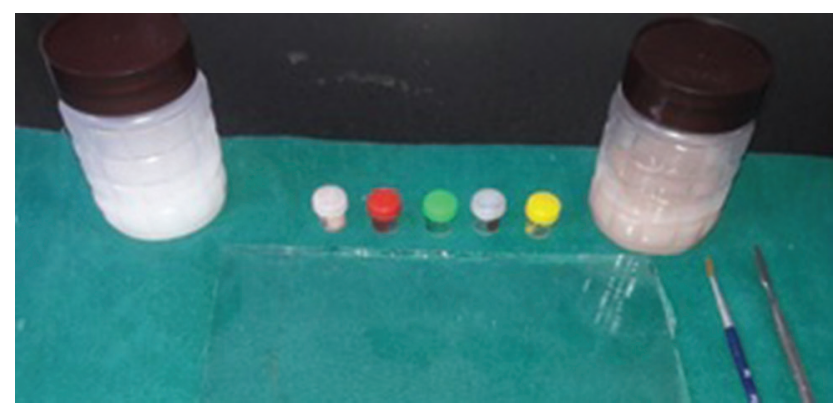

Fig. 7 Silicone elastomer and color pigments.

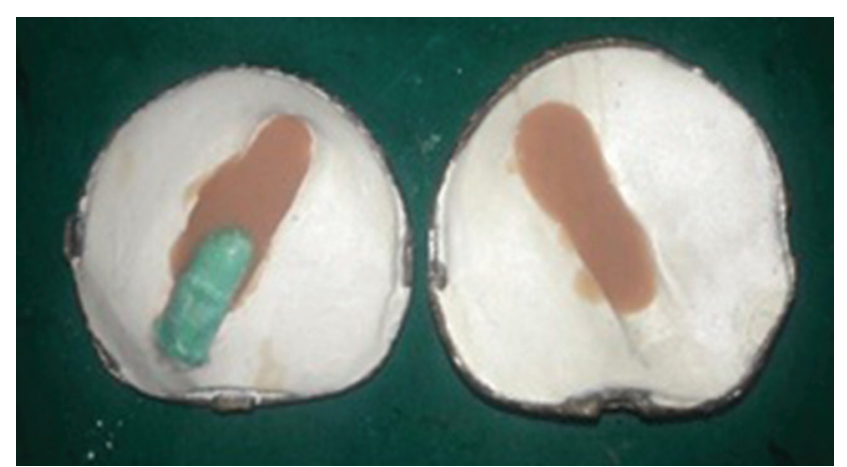

Fig. 8 Mold after de-waxing packed with silicone elastomer. 


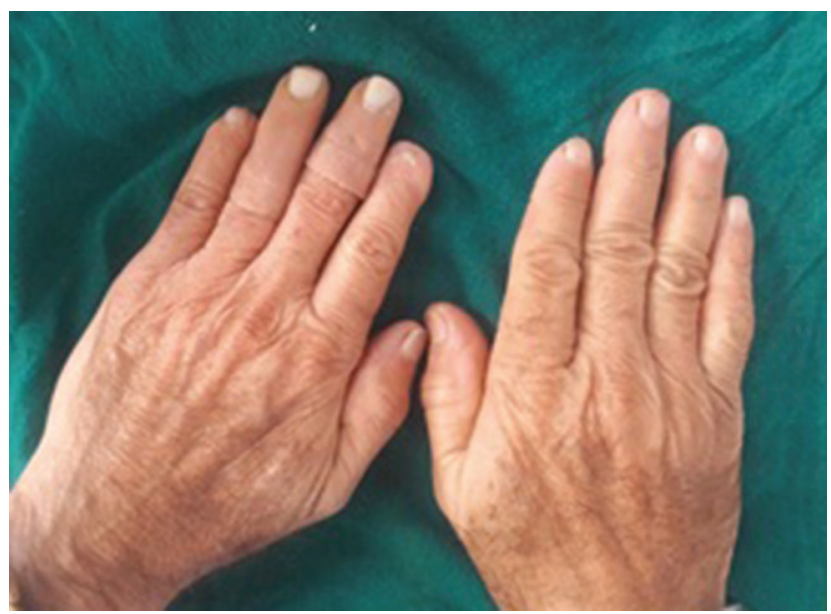

Fig. 9 Final prosthesis-dorsal.

check-up. Recall examination revealed healthy skin at the amputation site and good retention of the prosthesis. ${ }^{6}$

\section{Discussion}

Individuals who desire finger replacement usually have high expectations from their artificial prosthesis. The desire for esthetics is more in such patients. The polyvinyl chloride material earlier used has the disadvantage of permanent staining with ball point and newspaper ink and is not durable enough. Customized silicone prosthesis has a higher rate of acceptance in terms of comfort, durability, and stain resistance. Dragon Skin Medical Grade Silicone platinum cure was used for the fabrication of the prosthesis. It has high tear strength and high elongation, and is easy to pour with clear to translucent appearance along with good physical and electrical stability. It is a room temperature vulcanizing silicone and does not shrink on curing. Silicone prosthesis according to recent studies provides additional benefits like desensitization and protection of painful hypersensitive tissue at the amputation site by constantly putting a gentle pressure over the affected area. Various other methods of retention of the prosthesis are also available like using rings over the margins of the finger prosthesis, use of medical grade adhesive, or by implant retained prosthesis. ${ }^{4}$

\section{Conclusion}

The custom-made finger prosthesis is esthetically acceptable and comfortable for use in patients with amputated fingers, resulting in psychological improvement and regaining self-confidence and helps the patient to lead a healthy professional and social life. An esthetic and retentive prosthesis is the primary determinant factor in the successful prosthetic restoration of a finger. The retention in this case report was obtained by reducing the stump model and fabricating a thimble type prosthesis.

\section{Conflict of Interest}

None identified.

\section{References}

1 Shanmuganathan N, Maheswari MU, AnandkumarV,Padmanabhan TV, Swarup S, Jibran AH. Aesthetic finger prosthesis. J Indian Prosthodont Soc 2011;11(4):232-237

2 Leow ME, Prosthetist C, Pho RW. Optimal circumference reduction of finger models for good prosthetic fit of a thimble-type prosthesis for distal finger amputations. J Rehabil Res Dev 2001;38(2):273-279

3 Asnani P, Shivalingappa CG, Mishra SK, Somkuwar K, Khan F. Rehabilitation of amputed thumb with a silicone prosthesis. J Nat Sci Biol Med 2015;6(1):275-277

4 Jacob PC, Shetty KH, Garg A, Pal B. Silicone finger prosthesis. A clinical report. J Prosthodontics 2012;21(8):631-633

5 Kaira LS, Katna V. Glove type silicone finger prosthesis-a case report. Ind J Dent Sci 2012;4(2):40-41

6 Jain P, Arora G, Yadav A, Aggarwal S, Bhatnagar P. Fabrication of a glove type silicone finger prosthesis: a case report. Ann Dent Special Vol 2014;2(4):163 